Consórcio Setentrional de Educação a Distância Universidade de Brasília e Universidade Estadual de Goiás

Curso de Licenciatura em Biologia a Distância

\title{
SEBASTIÃO ADELSON ALVES
}

\section{AVALIAÇÃO DA GLICEMIA APÓS O USO DE GEL REPOSITOR ENERGÉTICO À BASE DE CARBOIDRATO}

BRASÍLIA - DF 


\title{
SEBASTIÃO ADELSON ALVES
}

\section{AVALIAÇÃO DA GLICEMIA APÓS O USO DE GEL REPOSITOR ENERGÉTICO À BASE DE CARBOIDRATO}

\begin{abstract}
Monografia apresentada, como exigência parcial para a obtenção do grau pelo Consórcio Setentrional de Educação a Distância, Universidade de Brasília/Universidade Estadual de Goiás no curso de Licenciatura em Biologia a distância.
\end{abstract}

\section{BRASÍLIA/DF}


SEBASTIÃO ADELSON ALVES

\section{AVALIAÇÃO DA GLICEMIA APÓS O USO DE GEL REPOSITOR ENERGÉTICO À BASE DE CARBOIDRATO}

Trabalho de Conclusão de Curso apresentado como exigência parcial para a obtenção do grau de Licenciado em Biologia do Consórcio Setentrional de Educação a Distância, Universidade de Brasília/Universidade Estadual de Goiás.

Aprovado em 11 de junho de 2011

Prof. Msc. Paula Marcela Duque Jaramillo

Universidade de Brasília

Orientadora

Prof. Msc.Lélia Leoi Romeiro

Avaliador I

Prof. Msc. Melissa Monteiro

Avaliador II

\section{BRASÍLIA/ DF}




\section{DEDICATÓRIA}

À minha família pelo apoio na realização deste trabalho. 


\section{AGRADECIMENTOS}

Agradeço a Deus por dar-me a graça de realizar mais um sonho.

Agradeço à minha família: Lúcia, Débora e Artur pela paciência e compreensão.

Agradeço à professora Paula Marcela Duque Jaramillo por aceitar ser minha orientadora e ter contribuído muito para a realização deste trabalho.

Agradeço a todos os professores do curso e em especial à professora Anne Caroline dias Neves pela dedicação, incentivo e por muitas vezes estreitar essa relação tão importante entre mestres e alunos.

Aproveito a oportunidade para agradecer o professor Éderson Oliveira, pelos conhecimentos transmitidos e por várias vezes me receber com sabedoria e paciência nos momentos em que eu mais precisei.

Aos atletas do clube de corrida da Academia Boca que acreditaram neste projeto e fazem parte importante deste sucesso.

Aos meus amigos de curso que foram muito importantes nessa jornada em busca do conhecimento. 


\section{RESUMO}

Este trabalho teve como objetivo avaliar o nível glicêmico ou a taxa de açúcar no sangue a um grupo de pessoas que praticam corrida de rua pelo menos 03 (três) vezes por semana. Foram coletadas amostras de sangue e medida a glicemia em dois tempos: no início e no final da corrida, sendo que na primeira coleta não foi feita nenhuma ingestão de carboidrato durante o tempo da corrida. Na segunda coleta, após 30 minutos de corrida, todos os participantes ingeriram um gel repositor energético à base de carboidrato. Após as medições se concluiu que o gel promoveu um aumento significativo na taxa glicêmica da maioria dos participantes, ficando a sugestão para que novas pesquisas sejam realizadas principalmente da sua aplicabilidade de acordo com o objetivo de cada atleta.

Palavras - chave: Metabolismo. Glicemia. Carboidrato. 


\section{SUMÁRIO}

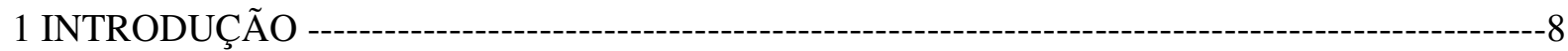

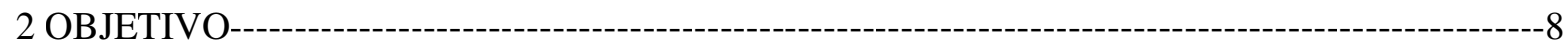

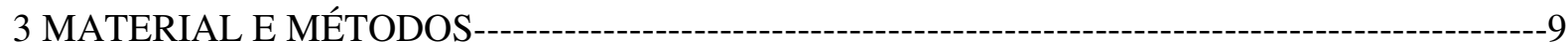

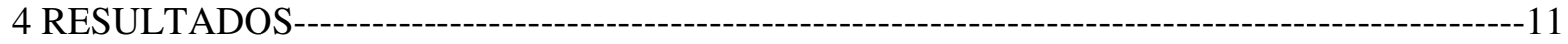

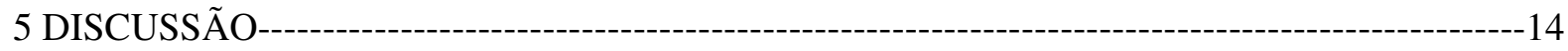

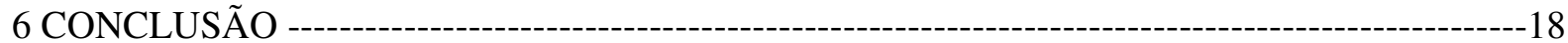

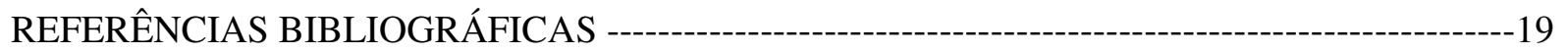


As Ciências Biológicas têm como objetivo o estudo dos seres vivos e suas funções vitais como respiração, circulação dentre outras. Os seres vivos estão em constante atividade e para que isso ocorra precisam produzir e gastar energia e essa produção tem como fonte os alimentos que funcionam como combustível. Todo esse processo que ocorre no organismo é chamado de metabolismo.

A bioquímica é uma parte da biologia que estuda, entre outras, gordura, sais minerais, carboidratos e suas ações no organismo. O carboidrato é uma das principais fontes de energia na forma de glicose no organismo, tendo como referência a taxa de açúcar no sangue (glicemia) e seu 11 comportamento em uma situação fora dos padrões de normalidade, por exemplo, em uma atividade 12 física de longa duração como uma corrida.

Existem hoje no comércio vários suplementos à base de carboidratos, oferecidos na forma líquida, em pó e em gel, com o propósito de repor o nível de glicemia ou taxa de açúcar no sangue, em situações em que haja a necessidade de mantê-la em níveis aceitáveis para que não comprometa o equilíbrio do indivíduo.

\section{OBJETIVO} glicêmica antes e depois de uma corrida com duração de uma hora em situações diferentes, sobre o uso de repositor energético em gel à base de carboidrato. 
O método utilizado e o tratamento dado aos resultados desta pesquisa foi o estudo de caso, que caracteriza-se principalmente pelo estudo do concentrado de um único caso. Esse tipo de estudo é preferido pelos pesquisadores que desejam aprofundar seus conhecimentos a respeito de um determinado caso específico (BEUREN et al., 2006).

O grupo de amostragem foi composto por 15 (quinze) pessoas, entre homens e mulheres com idade superior a 35 anos, que não apresentam nenhuma limitação física, complicação de saúde, como diabetes ou obesidade avaliado pelo índice de massa corporal (IMC), que possa alterar os resultados dos testes. Os participantes da pesquisa fazem parte de um grupo de corrida orientado por profissionais das áreas de saúde e educação física, há mais de 02 (dois) anos. Os testes foram aplicados no Parque Sara Kubitschek localizado na Asa Sul de Brasília, entre os dias 14 a 26 de março de 2011 no período noturno de acordo com a disponibilidade do grupo.

O produto a ser usado no teste foi o gel repositor energético que consiste de um sachê em porção única de $(30 \mathrm{~g})$ com $80 \mathrm{kcal}$, tendo em sua composição carboidrato $(20 \mathrm{~g})$, água, xarope de frutose, cloreto de sódio $(11 \mathrm{mg})$, vitamina C (4,2mg), vitamina E $(0,90 \mathrm{mg})$, acidulante ácido cítrico, aromatizante e conservantes sorbato de potássio $(4,4 \mathrm{mg})$ e benzoato de sódio, valores diários com base numa dieta de 2000kcal conforme dados do próprio produto expresso na embalagem. A escolha da marca do produto foi baseada na preferência do grupo.

$\mathrm{Na}$ primeira etapa não houve ingestão de suplemento, funcionando como controle. Foram coletadas amostras de sangue no início e no final do teste, perfurando o dedo anelar da mão com agulhas descartáveis para o teste de glicemia. Todos os procedimentos foram feitos por uma auxiliar de saúde devidamente credenciada e utilizando equipamentos de proteção individual.

$\mathrm{Na}$ segunda etapa, após 30 minutos de corrida, foi feita ingestão de um gel composto de carboidrato e outras substâncias com função de repositor energético. No início e no final da corrida foram coletados amostras de sangue para realização dos testes de glicemia de cada corredor para posterior análise. Foram registrados na ficha individual do participante além da idade, nome, peso e altura para um posterior cálculo de IMC, informação sobre a última refeição realizada devido à possibilidade de algum participante estar fazendo algum tipo de dieta rigorosa.

O aparelho e fitas utilizados para realização dos testes glicêmicos foram da marca AccuChek Active Monitor e os resultados dados em mg/dL, apresentados aqui através de tabelas e gráficos.

Utilizou-se o IMC ou índice de Quelet, que é calculado pelo peso corporal (kg) dividido pela altura em $\left(\mathrm{m}^{2}\right)$ e posteriormente correlacionado seu resultado com padrões pré-estabelecidos e 


\section{8}

89

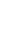

sugeridos pela Organização Mundial de Saúde (OMS-1997), para identificar algum possível caso de obesidade no grupo:

\title{
Classificação do IMC:
}

Magreza de grau $-3 \quad<16,0$

Magreza de grau $-2 \quad 16,1$ a 16,9

Magreza de grau - 1

Normal

18,5 a 24,9

Pré-obeso

25,0 a 29,9

Obesidade de grau - 1

30,0 a 34,9

Obesidade de grau - 2

35,0 a 39,9

Obesidade de grau -3

$>40,0$

\begin{abstract}
A glicemia foi avaliada com base nos resultados utilizados para teste em jejum, fornecido pelo Ministério da Saúde (MS) sendo levado em consideração que o objetivo deste trabalho não era identificar possíveis casos de diabetes, por isso deixando de ser relevante o horário e condições de alimentação:
\end{abstract}

\section{Normal}

Até $100 \mathrm{mg} / \mathrm{dL}$

\section{Elevado}

$100-139 \mathrm{mg} / \mathrm{dL}$

\section{Diabetes}

Mairo de $140 \mathrm{mg} / \mathrm{dL}$

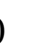

1

2

(1999) a coleta de dados constitui uma etapa importantíssima da pesquisa de campo, pois ocorre uma elaboração e análise das informações coletadas que são representadas graficamente facilitando a discussão dos resultados com base na análise e interpretação dos pontos de vista.

5

6
glicêmico em mais de $87 \%$ dos participantes $(n=13)$ (tabela 1 e gráfico 1). Os dados apresentaram uma média de $11,00 \mathrm{mg} / \mathrm{dl}$ entre os resultados obtidos dos avaliados, e desvio padrão (DP) = 5,2. Para avaliar o desvio padrão foram dispensados os dois resultados maiores e os dois menores das 
amostras da glicemia, colhidas no início e no final dos testes, tendo vista a variação apresentada por eles ser muito alta ou muito baixa, não sendo possível explicar nessa pesquisa essas alterações,

101 podendo vir a influenciar negativamente no desvio padrão e comprometer a pesquisa.

102

Tabela 1: Primeiro teste realizado sem o uso do gel energético repositor.

\begin{tabular}{|c|c|c|c|}
\hline Participantes & $\begin{array}{c}\text { Resultado antes da } \\
\text { corrida } \\
\text { (taxa de glicemia) }\end{array}$ & $\begin{array}{c}\text { Resultado após a } \\
\text { corrida } \\
\text { (taxa de glicemia) }\end{array}$ & $\begin{array}{c}\text { Resultados para } \\
\text { análise }\end{array}$ \\
\hline 01 & 91 & 75 & 16 \\
\hline 02 & 88 & 104 & -16 \\
\hline 03 & 130 & 87 & 43 \\
\hline 04 & 87 & 95 & -8 \\
\hline 05 & 103 & 88 & 15 \\
\hline 06 & 97 & 87 & 10 \\
\hline 07 & 97 & 82 & 15 \\
\hline 08 & 102 & 88 & 14 \\
\hline 09 & 91 & 63 & 28 \\
\hline 10 & 103 & 97 & 8 \\
\hline 11 & 90 & 82 & 5 \\
\hline 12 & 87 & 82 & 20 \\
\hline 13 & 95 & 75 & 7 \\
\hline 14 & 88 & 81 & 5 \\
\hline 15 & 95 & 80 & \\
\hline
\end{tabular}

104

105

106

107

108

109

110

111

112

113

114

115

116

117

118

119

120

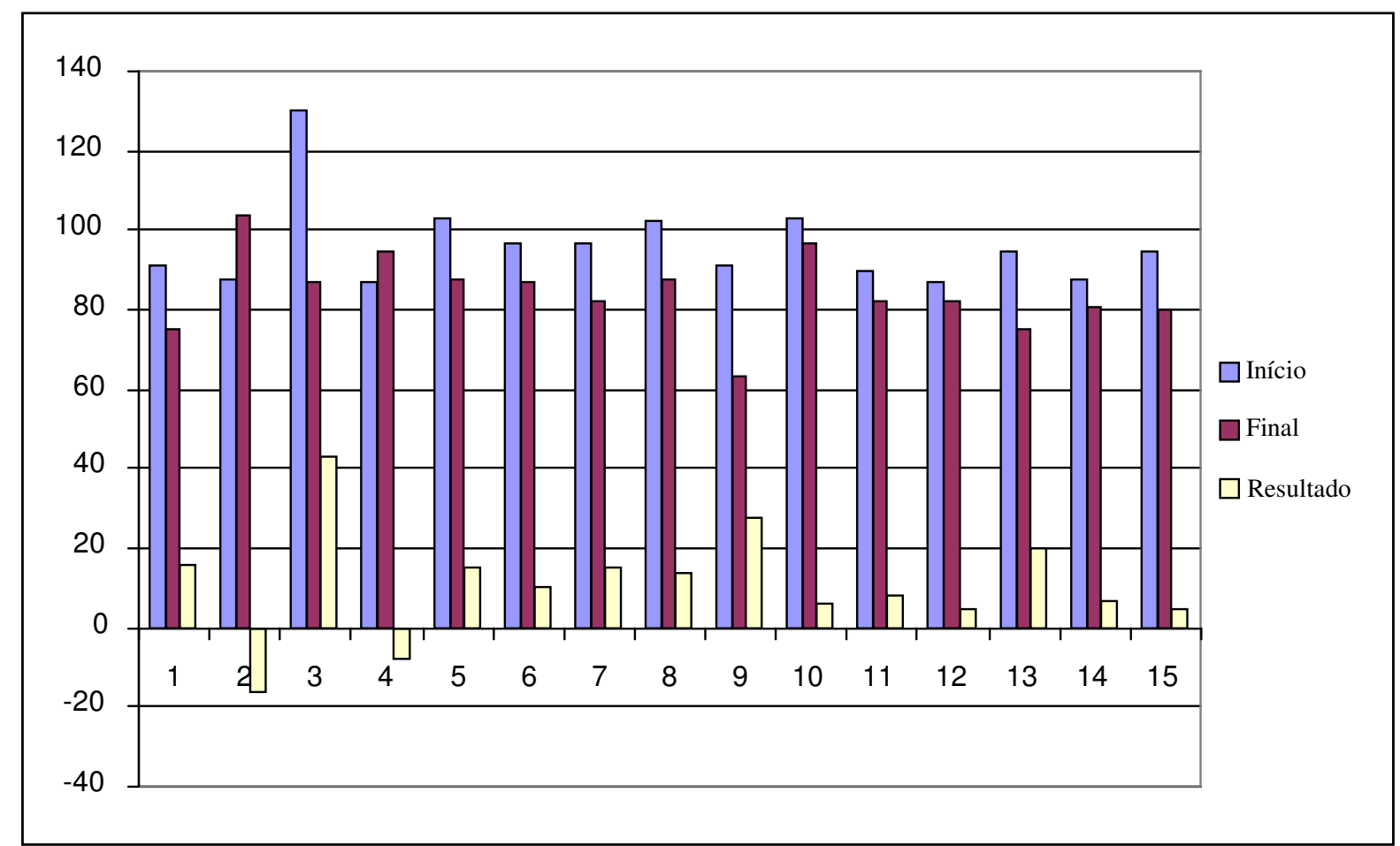

Gráfico 1: primeiro teste realizado sem o uso de gel energético repositor. 
Os resultados apresentados na tabela e gráfico 2, demonstram que cerca de $67 \%$ dos casos

122 do grupo pesquisado $(\mathrm{n}=11)$, houve aumento no nível da taxa glicêmica. Os resultados obtidos pela

123 diferença entre os valores obtidos no início e final dos testes glicêmicos, apresentaram uma média

124 de 13,13 mg/dl de aumento da taxa glicêmica, com um desvio padrão(DP) de 12,05. Para obter o

125 valor do DP foram dispensados os 02 (dois) valores maiores e menores dos resultados, buscando se

126 aproximar mais da realidade do grupo em termos de variação, para que não houvesse riscos para

127 pesquisa.

128

129 Tabela 2: Segundo teste realizado com o uso do gel energético repositor.

\begin{tabular}{|c|c|c|c|}
\hline Participantes & $\begin{array}{c}\text { Resultado antes da } \\
\text { corrida } \\
\text { (taxa de glicemia) }\end{array}$ & $\begin{array}{c}\text { Resultado após a } \\
\text { corrida } \\
\text { (taxa de glicemia) }\end{array}$ & $\begin{array}{c}\text { Resultados para } \\
\text { análise }\end{array}$ \\
\hline 01 & 91 & 88 & -3 \\
\hline 02 & 136 & 136 & 0 \\
\hline 03 & 112 & 97 & -15 \\
\hline 04 & 97 & 88 & 34 \\
\hline 05 & 107 & 141 & 3 \\
\hline 06 & 98 & 101 & 55 \\
\hline 07 & 97 & 152 & 22 \\
\hline 08 & 90 & 112 & 10 \\
\hline 09 & 93 & 103 & -6 \\
\hline 10 & 103 & 97 & 28 \\
\hline 11 & 87 & 115 & 29 \\
\hline 12 & 90 & 119 & 14 \\
\hline 13 & 87 & 101 & 22 \\
\hline 14 & 91 & 98 & 110 \\
\hline 15 & 88 & & \\
\hline
\end{tabular}

130

131

132

133

134

135

136

137

138

139

140

141

142 
143

144

145

146

147

148

149

150

151

152

153

154

155

156

157

158

159

160

161

162

163

164

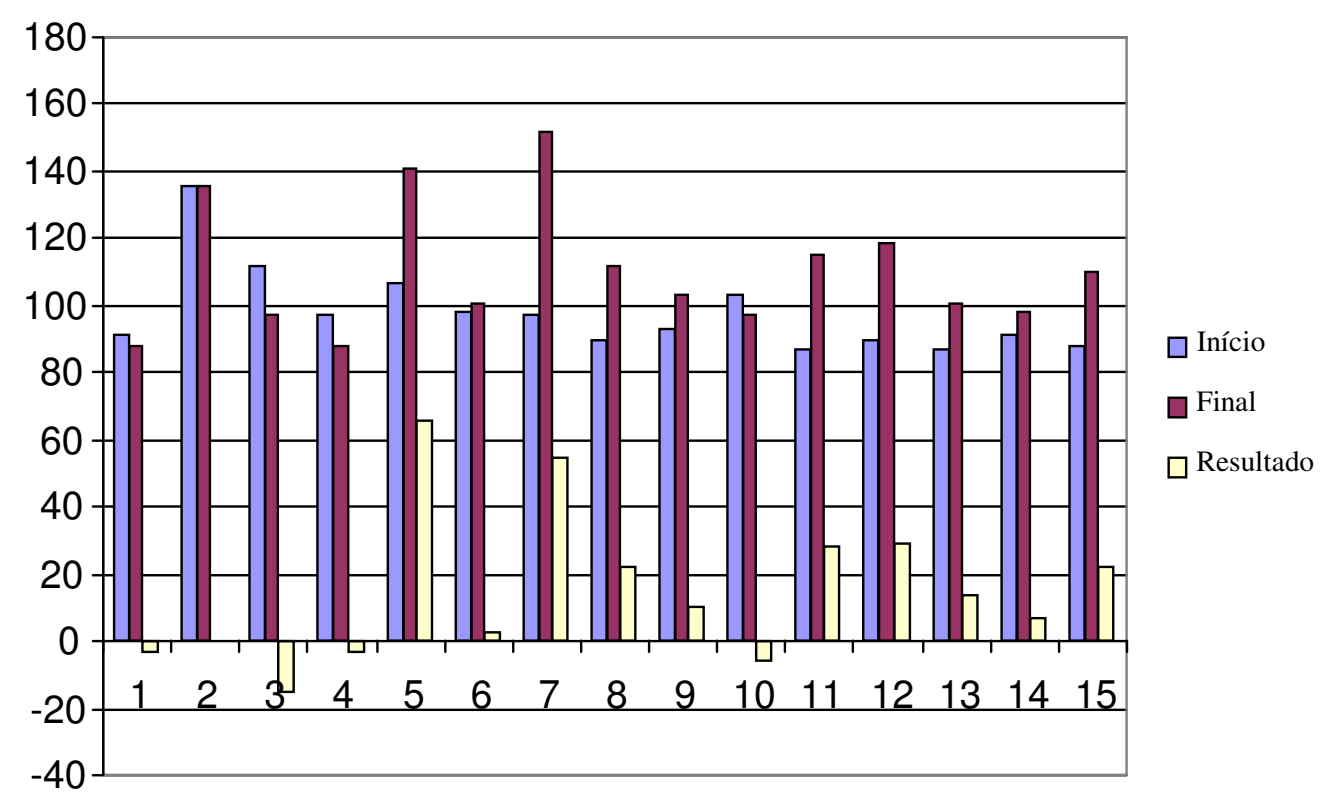

Gráfico 2: segundo teste realizado com uso do gel energético repositor

O IMC dos participantes apresentou variações entre 20 e 28 , apresentando casos de sobrepeso, demonstrando que a pesar de estarem em constante atividade física, em alguns casos o metabolismo é lento para queima de gordura, padrões que se considera dentro da normalidade ou que não possuem uma dieta alimentar adequada. (1) 
As variações ocorridas podem ter relação com a ação dos hormônios insulina e glucagon, que fazem a regulação da glicemia do sangue e das células. Segundo (McARDLE, 1992), a insulina promove o transporte de glicose do sangue para o interior das células hepáticas, enquanto o glucagon remove essa glicose do fígado e lança no sangue, exercendo o efeito hiperglicemiante.

171 Como a insulina, o glucagon é controlado pelo nível de glicose no sangue. Quando em um exercício

172 prolongado a glicose cai, as células pancreáticas secretam o glucagon, que irá liberar a glicose do

173 fígado para normalizar a glicemia, essa ação torna-se muito importante para o equilíbrio da glicose 174 durante o exercício.

175 Atualmente vários pesquisadores discutem sobre a importância da reposição hídrica e de se 176 fazer ingestão de carboidratos, antes e durante a atividade física de longa duração, com o objetivo 177 de melhorar o desempenho e prevenir possíveis prejuízos à saúde, em decorrência da desidratação e diminuição da taxa de glicose no sangue.

Os carboidratos são considerados a fonte primária de energia metabólica para o organismo, uma vez que seu catabolismo é um grande liberador de energia química, fornecendo essencialmente combustível para o cérebro, medula, nervos periféricos e células vermelhas do sangue (ROMBALDI, 1996), por isso sua importância durante a prática de uma atividade que envolva gasto energético elevado.

Aoki (2003), afirma que a suplementação de carboidrato é eficiente para o aumento do desempenho físico, e o mecanismo proposto para isso é a manutenção da glicemia e a redução da taxa de glicogenólise (quebra da glicose) atenuando assim, distúrbios homeostáticos como a hipoglicemia, que é um fator limitante para que se tenha um bom desempenho e se consiga atingir uma meta dentro de um treinamento visando atingir um objetivo.

Segundo Bertolucci (2002) a alimentação e hidratação inadequada dos praticantes de atividade física afetam diretamente seu rendimento e sua saúde, o peso e sua composição corporal, além de prejudicarem a disponibilidade de substratos durante o exercício e recuperação. De acordo com a Sociedade Brasileira de Medicina do Esporte (SBME), a ingestão de água e hidroeletrolíticos é fundamental nos primeiros sessenta (60) minutos de exercício, quando ultrapassa esse tempo é recomendável o uso de produtos que possuam carboidrato em sua constituição.

Para Maughan (2004) os suplementos nutricionais são consumidos com o objetivo de melhorar a saúde e prevenir doenças. Estes podem ser definidos como produtos feitos de vitaminas, minerais, produtos herbais, extratos de tecidos, proteínas, aminoácidos e outros produtos. 
suplementos de vitaminas e minerais cujas doses situam-se acima dos 100\% Dietary Reference Intakes (DRI) são considerados medicamentos, assim como os fitoterápicos. O termo suplemento acaba por não ter uma definição que facilite sua regulamentação, devido seu uso ser para vários produtos.

A portaria do Ministério da Saúde $\mathrm{n}^{\circ} 222$ Agência Nacional de Vigilância Sanitária (ANVISA) (www.anvisa.gov.br/legis/portarias/222_98.htm) define como repositor energético como sendo "produtos formulados com nutrientes que permitam o alcance e/ou manutenção do nível apropriado de energia para atletas". Segundo Hirschbruch (2008), os nutrientes energéticos presentes na formulação devem constituir no mínimo $90 \%$ de carboidratos, e são encontrados em barra, em pó, líquido ou em gel, opcionalmente podem conter vitaminas e /ou minerais.

O organismo está constantemente utilizando glicose como fonte energética por excelência, quando não é usada como combustível, é armazenada como glicogênio e gordura. A glicose sanguínea é considerada normal quando oscila entre 80 e $100 \mathrm{mg} / \mathrm{dl}$ no sangue venoso, após jejum de algumas horas. Em condições normais, existe um equilíbrio entre o fornecimento e o consumo de glicose nos períodos imediatamente após a alimentação, havendo uma tendência a elevar o nível de glicose, que tende a diminuir por volta de duas horas e meia após, retornando as cifras basais de jejum. A produção e utilização da glicose são reguladas pela alimentação, consumo periférico, presença de enzimas, insuficiências hormonais e nervosas (ARDUINO,1980).

A glicose $\left(\mathrm{C}_{6} \mathrm{H}_{12} \mathrm{O}_{6}\right)$ obtida através dos alimentos ricos em carboidratos, que embora seja a principal fonte de energia corporal é muito pouco armazenada como reserva de combustível, sendo seu excesso armazenado na forma de gordura. A glicose é encontrada sobre a forma de glicogênio, isto é, polímero de glicoses que correspondem a cerca de 430 gramas em um individuo de $70 \mathrm{~kg}$. A glicose está armazenada em forma de glicogênio nos músculos e no fígado, com pequenas quantidades encontradas nos demais tecidos. Existem diversas enzimas que fazem a conversão da glicose em glicogênio, o primeiro passo é a transferência de um grupo fosfato para a glicose advindo da hidrólise (quebra) de ATP (Adenosina Trisfosfato - forma de energia corporal) formando glicose-6-fosfato. Nesta forma, poderá ser degradada e ressintetizar o ATP ou formar o glicogênio (gliconeogênese), que ocorre quando um excesso de glicose está disponível para as células hepáticas ou musculares, feitas pelas enzimas sintéticas alostéricas que regulam a ativação e a inibição do trânsito de glicose (VANDER, apud, ALVES, 2000).

As células musculares esqueléticas não possuem essas enzimas que catalisam a remoção de fosfato para formar glicose livre, que pode passar pela membrana celular. Assim a glicose armazenada como glicogênio não pode ser liberada para outras células. Essa quantidade armazenada servirá como fonte emergencial de energia para as próprias células musculares, quando 
234 a taxa de glicose que lhes é liberada pelo sangue não for suficiente em uma intensa atividade 235 contrátil.

236 Através da glicólise, que é um processo metabólico que corre na ausência de oxigênio e 237 como consequência há formação de ácido pirúvico e ácido láctico, que poderá ser metabolizado 238 como combustível ou tamponado. As células hepáticas por outro lado contém enzimas que convertem o glicogênio em glicose livre que é liberdade da célula para o sangue desempenhando um papel muito importante na manutenção da glicose sanguínea (VANDER,1981)

$\mathrm{Na}$ transição do repouso para um exercício sub-máximo $(60 \%$ a $70 \%$ da capacidade aeróbica máxima), quase toda energia é fornecida pelo glicogênio acumulado nos músculos ativos durante os vinte minutos subsequentes, o glicogênio hepático e muscular proporciona cerca de $40 \%$ a 50\% da demanda energética, o restante desta demanda é proporcionada pelo metabolismo das gorduras, finalmente, a produção de glicose pelo fígado não consegue acompanhar a utilização pelo músculo, provocando a queda na glicemia a níveis hipoglicêmicos (menor que 45mg/100ml de sangue) após 90 minutos de exercícios contínuo provocando sensações de fraqueza, fome e vertigens, podendo causar danos cerebrais irreversíveis (McARDLE,1992). durante uma prova, se realmente a atividade tiver uma intensidade e duração capazes de levar a uma hipoglicemia. Para a grande parte das pessoas, isto se verifica em provas que excedem a três horas 252 de duração. 

de pessoas após o uso de repositor energético à base de carboidrato, esta pesquisa apresentou resultados significativos, pois a taxa glicêmica após a corrida sofreu aumento na maioria dos avaliados que fizeram uso do repositor. Devendo-se considerar que trata-se de um estudo preliminar de graduação, respeitando os limites deste pesquisador.

Sobre os testes e os resultados apresentados, não se pode afirmar com toda certeza que tal fato da elevação tenha ligação direta com o uso de gel repositor energético, pois conforme as literaturas que tratam da glicemia citadas, existe o metabolismo da glicose, aonde os hormônios insulina e glucagon agem diretamente no organismo para manter a glicose sanguinea dentro dos padrões de normalidade. Tendo em vista a mostra ser pequena e o metabolismo variar de uma pessoa para outra, recomenda-se que novos trabalhos sejam feitos e publicados para que tenhamos 283 
ALVES, Sebastião Adelson. Efeito agudo e crônico de um programa de atividade física em diabéticos idosos. Monografia de especialização apresentada à Universidade de Brasília. Brasília, DF, 2000.

AOKI MS, Pontes Junior FL, Navarro F, Uchida MC, Bacurau RFP. Suplementação de carboidrato não reverte o efeito deletério do exercício de endurance sobre o subsequente desempenho de força. Rev Bras Med Esporte. 2003; 9(5): 282-7.

ARDUINO, F. Diabetes mellitus. Rio de Janeiro: Guanabara Koogan, 1990.

ASSOCIAÇAO BRASILEIRA DE NORMA TÉCNICAS. NBR 6023: informações e documentação: referências : elaboração. Rio de Janeiro, maio 2002.

\section{Janeiro, 2002.}

.NBR 6034: informações e documentos: citações em documentos: apresentações. Rio de Janeiro, 2005.

BRASIL. Ministério da saúde. Secretária de Vigilância Sanitária . Portaria n ${ }^{\circ} 222$, de 24 de março de 1998. <www.anvisa.gov.br/legis/portarias/222_98.htm>. Acesso em: 10 de abril de 2011.

BEUREN, Ilse Maria, et al. Como Elaborar Trabalhos Acadêmicos em Contabilidade: teoria e prática. 3. ed. São Paulo: Atlas, 2006.

HIRSCHBRUCH, M. D. et al. Consumo de suplementos por jovens freqüentadores de academias de ginástica em São Paulo. Rev. Bras. Med Esporte. Niterói, v. 14, n. 6, p. 539-543, nov./dez. 2008.

MAUGHAN, R.J.; BURKE, L.M. Nutrição Esportiva. Artmed editora. Porto Alegre, 2004.

MCARDLE, W.D., KATCH, F.I. e KATCH, V. L. Fisiologia do exercício: energia, nutrição e desempenho humano. Rio de Janeiro: Guanabara Koogan, 1992.

NOVAES, Jeferson S. e VIANA, Jeferson M.- Personal training e condicionamento físico em academia. Rio de Janeiro: Shape, 1998.

ROGATTO, GP. Hidratos de carbono: aspectos básicos e aplicados ao exercício físico. Revista Digital - Buenos Aires. v. 8. p. 1-13. 2003.

ROMBALDI A.J. Alguns efeitos bioquímicos da ingestão de carboidrato na realização de trabalho intermitente de alta intensidade em ratos. Tese de doutorado apresentada à Universidade Federal de Santa Maria. Santa Maria, RS, 1996.

VANDER, A. J., SHERMAN, J. H. E LUCIANO, D. S. Fisiologia humana. São Paulo: Mcgrau350 hill, 1981. 